\title{
Type la Supernova: Observations and Theory
}

\author{
Jordi Isern* \\ Institute for Space Sciences (CSIC-IEEC) \\ E-mail: iserneieec.cat
}

\section{Eduardo Bravo}

Department of Nuclear Physics (UPC)/IEEC

E-mail: eduardo.bravo@upc.edu

\section{Alina Hirschmann}

Institute for Space Sciences (CSIC-IEEC)

E-mail: alina@ieec.cat

\begin{abstract}
There is a wide consensus that Type Ia supernovae are the outcome of the thermonuclear explosion of a carbon-oxygen white dwarf in a binary system. Nevertheless, the nature of this system, the process of ignition itself and the development of the explosion continue to be a mystery despite the important improvements that both, theory and observations, have experienced during the last years. Furthermore, the discovery of new events that are challenging the classical scenario forces the exploration of new issues or, at least, to reconsider scenarios that were rejected at a given moment.
\end{abstract}

11th Symposium on Nuclei in the Cosmos

19-23 July 2010

Heidelberg, Germany.

\footnotetext{
* Speaker.
} 


\section{Introduction}

Supernovae are characterized by a sudden rise of their luminosity, by a steep decline after maximum light that lasts several weeks, followed by an exponential decline that can last several years. The total electromagnetic output, obtained from the light curve, is $\sim 10^{49} \mathrm{erg}$, while the luminosity at maximum can be as high as $\sim 10^{10} \mathrm{~L}_{\odot}$. The kinetic energy of supernovae can be estimated from the expansion velocity of the ejecta, $v_{\exp } \sim 5,000-10,000 \mathrm{~km} / \mathrm{s}$, and turns out to be $\sim 10^{51} \mathrm{erg}$. Such amount of energy can only be obtained in two ways, from the gravitational collapse of an electron degenerate core to form a neutron star or a black-hole [1] or from the thermonuclear incineration of a carbon-oxygen degenerate core [2].

In both cases, the presence of a relativistic degenerate stellar core is necessary. This is a consequence of the fact that such cores are only weakly bound and do not have a definite length scale, which means that the injection or the removal of relatively small amounts of energy causes large variations of the radius of the star. Stellar degenerate cores may have the following different main compositions: helium, carbon plus oxygen, oxygen, neon and magnesium, and iron. Their thermonuclear explosion or gravitational collapse within different environments can produce very different astronomical phenomena. He-cores always explode because of the extreme flammability of the fuel. $\mathrm{CO}$ cores can explode or collapse depending on the ignition density and on the velocity of the burning front [3]. If the density is larger than $\sim 5.5 \times 10^{9} \mathrm{~g} / \mathrm{cm}^{3}$ and the flame is laminar they can collapse to a neutron star [4]. ONe cores ignite at such densities that they collapse [5] and Fe cores always collapse because of their inability to release nuclear energy.

From the spectroscopic point of view, Type Ia supernovae are characterized by the absence of hydrogen and the presence of a prominent Si II line at maximum light (in fact these are the characteristics that define the class). From the photometric point view they experience a sudden rise to maximum of light, $\left\langle M_{\mathrm{B}}>\sim-19^{\mathrm{mag}}\right.$, in 20 days or less, followed by a sudden decline of $\sim 3^{\mathrm{mag}}$ in 30 days and an exponential tail with a characteristic time $\sim 70$ days. From the point of view of the galaxies hosting supernovae, it was immediately realized that SNIa occur in all galaxy types in contrast with all the other supernova types that only appear in galaxies with recent star formation activity. It is interesting to notice here that the SNIa rate correlates with the star formation rate [6], indicating that the progenitors must be able to produce prompt and delayed explosions. All these arguments immediately led to the conclusion that SNIa were caused by the thermonuclear explosion of a CO white dwarf near the Chandrasekhar mass in a close binary system, an idea that was strongly supported by the spectrophotometric homogeneity displayed by this kind of supernovae [7,8].

Despite their remarkable homogeneity, when observed in detail some differences appear. On one hand there is a group with very bright and very broad peak that exhibits Fe III lines before the maximum. The prototype is SN1991T and represent 9\% of all the events. On the other hand there is a group with very dim and narrow peaks that lack of the characteristic secondary peak in the infrared. The prototype is SN1991bg and they represent the $21 \%$ of all the events. To these categories it has been recently added a new one that contains very peculiar supernovae. The prototype is SN2002cx and they represent the 4\% of the total. These supernovae are characterized by a high ionization spectral features in the premaximum, like the $91 \mathrm{~T}$ class, and by a very low luminosity and the lack of secondary maximum, like the $91 \mathrm{bg}$ class. The remaining ones, the $66 \%$, 
have a normal behavior and are known as Branch-normal. However, even the normal ones are not completely homogeneous and show different luminosities at maximum and light curves with different decline rates [?].

The spectroscopic observations at different epochs allow to obtain a tomography of supernovae. At maximum light, the spectrum is characterized by the lines of intermediate mass elements that move at high velocities ( $\mathrm{v} \sim 8,000-30,000 \mathrm{~km} / \mathrm{s}$ ) indicating that thermonuclear burning was not complete in the outer layers [8]. On the contrary, during the nebular phase the spectrum is dominated by iron peak elements [9]. This fact together with the decrease of the cobalt lines [10] gave support to the idea that the tail is powered by the disintegration of ${ }^{56} \mathrm{Co}[11,12]$. These observations indicated that, in order to avoid the total incineration of the outer layers, the burning front should propagate subsonically as a deflagration, while detonations should be limited to layers with densities smaller than $\sim 10^{7} \mathrm{~g} / \mathrm{cm}^{3}$. Consequently, several models combining subsonic and supersonic burning fronts have appeared in the literature. The basic ones are: pure deflagration [13] in which the front propagates subsonically all time; delayed detonation [14], in which the front starts subsonically and turns out into a detonation when the flame reaches a critical density, $\rho_{\mathrm{c}} \sim 2 \times 10^{6}$ to $5 \times 10^{7} \mathrm{~g} / \mathrm{cm}^{3}$; pulsating delayed detonation [15] in which the flame starts subsonically, the star expands, contracts and detonates. Finally, a different category based on the detonation of a CO white dwarf with a mass smaller than the Chandrasekhar's mass induced by the detonation of a freshly accreted He layer. With the increase of the computation power, all these models have been generalized to three dimensions [?]. The main difference with the one dimensional models is that the number of parameters describing the initial conditions increases noticeably. In one dimension the initial parameters are the radius at which the runaway starts and the properties of the white dwarf. In three dimensions it is also necessary to include the number and the size, position and velocity distribution of the spots that will induce the ignition of the star. Since the outcome of the explosions strongly depends on the initial conditions described by these parameters, it is necessary to carefully compute the evolution of the white dwarf prior the explosion.

Despite the substantial advances of the last years, basic questions like which systems explode, why they explode and how they explode have not been satisfactorily answered yet. Since the problem of the explosion itself is analyzed in detail by F. Röpke in this volume, we will limit ourselves to the questions of which are the progenitors and which is the origin of the width-luminosity relationship.

\section{Progenitors}

There is a wide consensus that the progenitors have to be composed by a $\mathrm{CO}$ white dwarf plus a close enough companion able to provide the mass necessary to trigger the thermonuclear instability, and several evolutionary paths leading to the instability have been identified [16]. The systems able to explode can be classified according to the nature of the donor (normal or degenerate stars, also known as SD or single degenerate secnarios and DD or double degenerate scenarios) and the composition of the accreted matter (hydrogen, helium or a mixture of carbon and oxygen).

The first systems identified as potential supernova progenitor were those formed by a white dwarf that accretes hydrogen [17]. There are many of them: cataclysmic variables, classical novae, recurrent novae, symbiotic stars and supersoft X-ray sources. 
If the accretion rate is smaller than $\sim 10^{-8}, 10^{-9} \mathrm{M}_{\odot} / \mathrm{yr}$, the accreted matter becomes degenerate and experiences a strong flash that can be identified with a nova. However, such events not only expel all the accreted mass but also erode the white dwarf preventing it from reaching the critical mass. The accreted mass can only be retained if $M_{\mathrm{WD}}>1.35 \mathrm{M}_{\odot}$, but white dwarfs with such initial masses are made of oxygen and neon and cannot explode as a SNIa. Thus, only white dwarfs that have experienced a previous accretion episode can reach the Chandrasekhar's mass at such rates. For intermediate rates $10^{-9} \leq \dot{M}_{\mathrm{H}}\left(\mathrm{M}_{\odot} / \mathrm{yr}\right) \leq 5 \times 10^{-7}$ hydrogen burns steadily or through mild flashes producing helium that accumulates. If the accretion rate is high enough, the freshly formed helium is converted into carbon and oxygen through weak flashes or steady burning and the white dwarf can approach to the Chandrasekhar mass. But if the accretion rate is roughly in the range $10^{-9} \leq \dot{M}_{\mathrm{H}}\left(\mathrm{M}_{\odot} / \mathrm{yr}\right) \leq 5 \times 10^{-8}$, the helium layer explodes under degenerate conditions and can trigger the explosion of all the star [18].

One of the problems posed by the SD scenario is that to avoid the constraint imposed by novae, the accretion rates have initially to proceed at a high rate and this makes white dwarfs hot and potentially detectable as X-ray sources. If it is assumed that all SNIa come from supersoft Xray sources, the number necessary to sustain the estimated rate of supernovae in M31 or the Milky Way is $\sim 10^{3}$ while current surveys have only detected $\sim 10-10^{2}$ [19]. In the case of elliptical galaxies the problem seems worst. At the first glance it seems that this argument favors the DD scenario but, to become a DD the system must evolve through two common envelope stages and this means that they will appear for some time, $>10^{6} \mathrm{yr}$, as symbiotic stars with a white dwarf burning hydrogen at the surface. It is therefore clear that the problem lies on the observational properties of these high rate accreting sources [20].

A possible way out of this problem could consist on the formation of heavy winds able to hide the nuclear burning white dwarf, as well as to consider variable accretion rates able to produce successively symbiotic stars, supersoft X-ray sources and, when the white dwarf is massive enough, recurrent novae. In any case, plausible evolutionary paths have been advanced [21]. Minor possible objections could come from the non detection of the hydrogen stripped from the companion by the explosion and the non-detection of the surviving star in the case of recent events in the Milky Way $[22,23]$

Close enough binaries formed by two intermediate mass stars can experience two episodes of common envelope evolution and form a double degenerate system made of two $\mathrm{C} / \mathrm{O}$ white dwarfs. The emission of gravitational waves induces a further reduction of the orbit and if the separation of both white dwarfs is not too large they eventually merge in less than a Hubble time [24, 25]. The main advantages of the DD scenario are that there are several known systems able to merge in a short time, although their mass is smaller than the critical mass, and that the time distribution of the persistent component of SNIa in galaxy clusters is only compatible with the merging of two white dwarfs [26]

During the merging process the secondary is destroyed in few orbital periods after filling the Roche lobe and forms a thick and hot accretion disk around the primary [27]. The impact is not able to induce a prompt ignition [28] and the outcome depends on the interaction between the disk and the white dwarf and the rate at which mass is transfered to the white dwarf. If the accretion rate is spherically symmetric, $\dot{M} \geq 2.7 \times 10^{-6} \mathrm{M}_{\odot} / \mathrm{yr}$ and the entropy of the infalling matter is neglected, the white dwarf ignites off center and becomes an ONeMg white dwarf [29] 
that eventually collapses to a neutron star [5]. Probably this approach is extremely simple as show the 3D models. Calculations indicate that a rotating hot corona forms and that the off-center ignition can be avoided if the following conditions are fulfilled: i) the ignition temperature is not reached at the interface when the quasi-static equilibrium is reached, ii) the time scale for neutrino cooling is shorter than the time scale for angular momentum dissipation, and iii) the mass accretion rate is $\leq 5 \times 10^{-6}, 10^{-5} \mathrm{M}_{\odot} / \mathrm{yr}$ [30]. It is obvious that the coupling between the disk and the rotating white dwarf is a critical issue [31, 32]. Furthermore, the recent analysis of SN2003fg [33] suggests that this type Ia supernova is about 2.2 times more luminous than the average. If the standard analysis is valid, the mass of nickel synthesized should be $\sim 1.3 \mathrm{M}_{\odot}$ and the ejected mass $\sim 2.1 \mathrm{M}_{\odot}$ in order to account for the spectrum at maximum, which implies that the exploding object should have a mass larger than the Chandrasekhar mass and the only way known to push the mass of a degenerate structure beyond the critical mass is through rotation, a natural outcome of the merging of two white dwarfs [31].

A possible issue to the problem posed by the explosion/collapse dilemma could be provided by the case of merging of two white dwarf of the same mass. In this case both stars maintain the individuality of their cores for some time and the final outcome is an explosion with the characteristics of subluminous SNIa [34]. Of course, the problem is the statistical relevance of the case.

There are two scenarios in which a white dwarf can directly accrete helium. One is a double degenerate with a secondary made of helium that merge as a consequence of the emission of gravitational waves, the other is a single degenerate scenario in which the secondary is a non-degenerate helium star and the mass transfer is powered by the helium burning. One dimensional models indicate that helium can ignite just above the base of the freshly accreted mantle under degenerate conditions. The high flammability of helium together with the low density of the envelope induces the formation of a detonation that incinerates the envelope and triggers the thermonuclear explosion of all the accretor despite the fact that its mass is smaller than the Chandrasekhar's mass [18].

Sub-Chandrasekhar models are not the favorites to account for the bulk of supernovae. The reason is that they predict the existence of a very fast moving layer made of ${ }^{56} \mathrm{Ni}$ and ${ }^{4} \mathrm{He}$ that is not observed [35]. However, if it was possible to neglect the contribution of the outer layer, these explosions could nearly reproduce the gross features of SN Ia explosions with a single parameter, the initial mass of the white dwarf [36]. In this sense it is important to realize that the process of formation of the mantle is quite complex and far from the simple description of matter accreted with spherical symmetry, at a constant rate and with the same entropy as the envelope, opening the possibility to trigger the detonation of the white dwarf without incinerating the outer He-layer [37]. Another fact to be taken into account is that in real systems like AM CVn binaries, the process of merging is not catastrophic and the accretion rate evolves with the mass of the donor, in such a way that helium is burned in a series of flashes that end with a "last-flash" that involves a mass of the order or slightly smaller than $0.1 \mathrm{M}_{\odot}$ that produces a dim SN Ia [38]. Possible counterparts of such events are SN2002Bk [39] and SN2005E [40], although other interpretations are possible.

\section{The width-luminosity relationship}

Type Ia are approximate standard candles since their dispersion at maximum is relatively small, $\sim 1.5^{\text {mag }}$, or even smaller if the sample is restricted to the "Branch-normals". Fortunately, 
there is a correlation between the brightness at maximum and the decline rate of the light curve just after this maximum [41] that allows to reduce the dispersion of brightness. This width-luminosity relationship (WLR) was definitively established from CCD measurements [42] and the dispersion of the light curves was reduced to values $\sim 0.15^{\text {mag }}$, converting SN Ia in true standard candles. It was believed that the dispersion of the calibrated magnitudes could be reduced to less than $0.1^{\text {mag }}$ but it is now clear that it is larger than the value expected from photometric errors only [43]. This relationship has proved to be extremely useful up to now, but the requirements of precision cosmology demand a higher precision as well as the certitude that the accuracy is not eroded by evolutionary effects at large distances.

It has been shown $[44,45]$ that the WLR is not primarily caused by differences in the diffusion time of photons but by evolutionary changes in the opacity/emissivity at the different photometric bands. They have shown that the FeII/CoII lines blanket the B-band and increase the emissivity at longer wavelengths, thus reducing the flux in the B-band. At temperatures larger than $\sim 7000$ $\mathrm{K}$, the FeII/CoII lines are absent or weak, the optical continuum behaves like a blackbody and the behavior of the color and the spectrum is almost independent of the mass of nickel, $M_{\mathrm{Ni}}$, synthesized. When the temperature goes below $\sim 7000 \mathrm{~K}$, Fe III recombine, the FeII/CoII lines become stronger and modify the behavior of the photometric bands. Since models with small values of $M_{\mathrm{Ni}}$ have smaller luminosities and temperatures, this effect appears more rapidly and produces a steeper B-band light curve. The result is that dim supernovae decline more rapidly than bright supernovae.

Although the Phillips relationship is closely related to $M_{\mathrm{Ni}}$, this parameter alone cannot account for the WLR and other factors like the total mass burned, the amount and distribution of stable iron, radioactive nickel and intermediate mass elements must combine in a quite tight way to produce this relationship $[45,46]$. However, some of the factors that shape the WLR depend on the evolution of the white dwarf prior to the explosion and project some shadows to the use of SNIa as as high precision distance indicators. For instance, the mass and distribution of stable iron depend on the density at which the ignition starts and, through the ${ }^{22} \mathrm{Ne}$ abundance, on the initial metallicity of the white dwarf $[47,48,49]$. Therefore, it is of critical importance to disentangle the role of these parameters to understand the limits of validity of the Phillips relationship. This is specially urgent since the discovery of the super-Chandrasekhar SNIa, that are very bright and apparently do not follow the WLR.

Certainly, the most important quantity is the amount of nickel synthesized and its distribution within the explosion debris. A first estimation can be obtained from the Arnett's law [50], however its precision is limited by the assumption of spherical symmetry and the use of the diffusion approximation. The nebular phase also provides the opportunity to obtain the value of $M_{\mathrm{Ni}}$, as well as the mass of inert iron, by accurately modelling the emission lines of the iron peak elements [46]. The problems come from the complexity of the NLTE models and the uncertainties of the atomic parameters.

The detection of the gamma-ray emission of radioactive elements produced during the explosion provides an independent and effective method to determine $M_{\mathrm{Ni}}$ [51]. In the SNIa case the dominant radioactive chains are ${ }^{56} \mathrm{Ni} \rightarrow{ }^{56} \mathrm{Co} \rightarrow{ }^{56} \mathrm{Fe}$ and ${ }^{57} \mathrm{Ni} \rightarrow{ }^{57} \mathrm{Co} \rightarrow{ }^{57} \mathrm{Fe}$. Differences in the amount of radioactive material, in the distribution within the ejecta as well as in the density, velocity and chemical composition profiles translate into the intensity and temporal evolution of the radioactive lines as well as into the importance and extension of the continuum component 
of the spectrum. This information allows to obtain not only the mass of the radioactive elements freshly synthesized but also some insight on the explosion mechanism [52, 53, 54, 55, 56]. The most important advantage of this method is that the calculations are relatively straightforward and that the opacities are better known in the gamma-ray domain than in other parts of the spectrum.

The $158,812 \mathrm{keV}{ }^{56} \mathrm{Ni}$-lines peak very early, near the maximum of light, and because of absorption, they are much weaker than those of ${ }^{56} \mathrm{Co}$. An early detection of these lines could provide information about the location of ${ }^{56} \mathrm{Ni}$ in the debris. The $847 \mathrm{keV}{ }^{56} \mathrm{Co}$-line reaches its maximum intensity roughly two months after the explosion and it is the most prominent one. At this moment, the debris have expanded so much that the intensity of the line is essentially determined by the total mass of the radioactive isotopes. Four months after the explosion, the ejecta are optically thin and the intensity of the lines is proportional to the total mass of the parent isotopes [54].

The main difficulty lies in the lack of sensitivity of current detectors in the region of $\sim 1 \mathrm{MeV}$. This lack of sensitivity is worsened by the fact that the SNIa radioactive lines are broad, of the order of 3 to $5 \%$. For instance the $\mathrm{W} 7$ model predicts a width of $35 \mathrm{keV}$ in the case of the 847 $\mathrm{keV}{ }^{56} \mathrm{Co}$ line. As a consequence of the noise, this fact reduces the sensitivity of the detector by a factor 3 to 4 as compared with the sensitivity of the narrow lines [54]. This means that current detectors only allow the measurement of $\mathrm{Ni} / \mathrm{Co}$ lines up to a distance of $\sim 8 \mathrm{Mpc}$, which reduces the possibility of observing SNIa to a few fortuitous cases.

The content of $M_{\mathrm{Ni}}$ depends on the supernova subtype. Roughly speaking it goes from more than $1 \mathrm{M}_{\odot}$ in the overluminous cases to a mere $0.08 \mathrm{M}_{\odot}$ in the extremely underluminous cases [46]. Therefore, this measurement will be useful only in the case it is obtained for a statistically relevant sample. A simple examination of the supernova catalogs indicates that to achieve this goal it is necessary to be able to detect supernovae up to a distance of $50 \mathrm{Mpc}$. Taking the W7 models as representative, this implies a sensitivity of $\sim 10^{-7} \mathrm{~cm}^{-2} \mathrm{~s}^{-1} \mathrm{keV}^{-1}$, value that is far form the performance of the current day detectors and demands the development of new concepts as could be the case of the Laue lens.

\section{Conclusions}

During the last years there has been a substantial advancement in our understanding of the origin and properties of Type Ia supernovae. Nevertheless, the most basic questions: which systems explode, why they explode and how they explode still remain unanswered.

Concerning the progenitors, two important points are the discovery of anomalous events and a preliminary determination of the delay time distribution in galaxy clusters. The existence of events that deviate from the astonishing homogeneity of SNIa allows to recover plausible scenarios, like the Sub-Chandrasekhar ones, that were initially rejected because they were unable to fit the observations. This fact provides the opportunity to obtain insight on the behavior of strongly interacting binary systems that contain one or two white dwarfs. The temporal distribution of explosions in the clusters of galaxies, together with the correlation of the SNIa rate with the star formation rate strongly suggests that the SDs and DDs scenarios can coexist. Numerical simulations in three dimensions of the behavior of the merged white dwarfs indicates that some of the hypothesis introduced in one dimensional models could be critical in order to understand the process of ignition and should be revised. 
Finally, given the prominent role played by the amount of nickel synthesized during the explosion, this quantity should be independently determined with a more robust method. Probably the detection of the $\gamma$-ray emission from ${ }^{56} \mathrm{Ni}$ and ${ }^{56} \mathrm{Co}$ would be the best issue.

\section{Acknowledgments}

This work has been supported by the MICINN grant AYA08-1839/ESP, by the ESF EUROCORES Program EuroGENESIS (MICINN grant EUI2009-04170), by the 2009SGR315 of the Generalitat de Catalunya and EU-FEDER funds.

\section{References}

[1] F. Zwicky, On collapsed neutron stars, ApJ 88 (522) 1938

[2] F. Hoyle, W.A. Fowler, Nucleosynthesis in Supernovae, ApJ 132 (565) 1960

[3] R. Canal, J. Isern, J. Labay, The origin of neutron stars in binary systatems, ARAA 28 (183) 1990

[4] E. Bravo, D. García-Senz, Coulomb corrections to the equation of state of nuclear statistical equilibrium matter: implications for SNIa nucleosynthesis and the accretion induced collapse of white dwarfs, MNRAS 307 (984) 1999

[5] K. Nomoto, Y. Kondo, Conditions for accretion-induced collapse of white dwarfs ApJ 367 (L19) 1991

[6] M. Sullivan et al., Rates and properties of Type Ia supernovae as a function of mass and star formation in host galaxies ApJ 648 (868) 2006

[7] R. Cadonau, G.A. Tamman, A. Sandage, Type I supernovae as standard candles, in Spernovae as distance indicators, vol. 24 of Lecture Notes in Physics, Berlin Springer Verlag (151) 1985

[8] A.V. Filippenko, Optical spectra of supernovae, ARAA 35 (309) 1997

[9] W. Li et al., Nearby supernova rates from the LickObservatory Supernova Search, arxiV 1006.461 2010

[10] T. Axelrod, Late time optical spectra from the Ni-56 model for Type I supernovae, PhD Thesis University of California Santa Cruz 1980

[11] M. J. Kuchner, R. P. Kirshner, P. A. Pinto, B. Leibundgut, Evidence for Ni-56 yields Co-56 yields Fe-56 decay in Type Ia supernovae, ApJ 426 (L89) 1994

[12] J. W. Truran, W. D. Arnett, A.G. W. Cameron, Nucleosynthesis in supernova shock waves, Can. J. Phys. 45 (2315) 1967

[13] S. A. Colgate, C. McKee, Early supernova luminosity, ApJ 157 (623) 1969

[14] K. Nomoto, D. Sugimoto, S. Neo, Carbon deflagration supernovae, an alternative to carbon detonation Ap\&SS 39, (L37) 1976

[15] A.M. Khokhlov, Delayed detonation models for Tye Ia supernovae A\&A 245 (114) 1991

[16] A. M. Khokhlov, Nucleosynthesis in delayed detonation models of Type Ia supernovae A\&A $\mathbf{2 4 5}$ (L25) $1991 b$

[17] K.Nomoto, Accreting white dwarf models for Type I supernovae. II-Off-center detonation supernovae ApJ 257 (780) 1982 
[18] S.E. Woosley, T. A. Weaver, Sub-Chandrasekhar mass models for Type Ia supernovae ApJ 429 (300) 1994

[19] K. A. Postnov, L. R. Yungelson, The evolution of compact binary star systems astroph/0701059 2007

[20] J. Whelan, I.Iben, Binaries and supernovae of Type I ApJ 186 (1007) 1973

[21] R. Di Stefano, Progenitors of Type Ia supernovae. I Are they supersoft X-ray sources? ApJ 712 (728) 2010

[22] R. Di Stefano, The progenitors of Type Ia supernovae. II Are they double degenerate binaries? The symbiotic channel ApJ 719 (474) 2010

[23] I. Hachisu, M. Kato, K. Nomoto, Young and massive binary pogenitors of type Ia supernovae and their circumstellar matter ApJ 679 (1390) 2008

[24] P. Ruiz-Lapuente et al., The binary progenitor of Tycho Brahe's 1572 supernova Nature 431 (1069) 2004

[25] E. E. Kerzendorf et al., Subaru high-resolution of star G in the Tycho supernova remnant ApJ 701 (1665) 2009

[26] R. F.Webbink, Double white dwarfs as progenitors of R Coronae Borealis stars and Type I supernovae ApJ 277 (355) 1984

[27] I. Iben, V. A. Tutukov, On the evolution of close binaries with components of initial mass between 3 solar masses and 12 solar masses ApJS $\mathbf{5 8}$ (661) 1985

[28] D. Maoz, K. Sharon, A. Gal-Yam, The supernova delay time distribution in galaxy clusters and implications for Type Ia progenitors and metal enrichment, astroph/10063576

[29] W. Benz, A. G. W. Cameron, W. H. Press, R. L. Bowers, Dynamic mass exchange in doubly degenerate binaries. I-0.9 and 1.2 solar mass stars ApJ 348 (647) 1990

[30] J.Guerrero, E. Garcia-Berro, J. Isern, Smoothed particle hydrodynamics simulation of merging white dwarfs $A \& A \mathbf{4 1 3}$ (257) 2004

[31] K. Nomoto, I. Iben, Carbon ignition in a rapidly accreting degenerate dwarf.-A clue to the nature of the merging process in close binaries ApJ 297 (531) 1985

[32] S. C. Yoon, Ph.Podsiadlowski, S. Rosswog, Remnant evolution after a carbon-oxygen white dwarf merger MNRAS 380 (933) 2007

[33] L. Piersanti, S. Gagliardi, I. Iben, A. Tornambe, Carbon-oxygen white dwearf accreting CO-rich matter. II. Self-regulating accretion process up to the explosive stage ApJ 598 (1229) 2003

[34] H. Saio, K. Nomoto,Off-center ignition in rapidly rotating accreting carbon-oxygen white dwarfs ApJ 615 (444) 2004

[35] D. A. Howell et al., The type Ia supernova SNLS-03D3bb from super-Chandrasekhar mass white dwarf star Nature 443 (308) 2006

[36] R. Pakmor, M. Kromer, F. F. Ropke, S. A. Sim, A. J. Ruiter, W. Hillebrandt, Sub-luminous type Ia supernovae from the mergers of equal-mass white dwarf with 0.9 solar masses Nature 463 (61) 2010

[37] P. Hoeflich, A. Khokhlov, Explosion models for type Ia supernovae: acomparison with observed light curves, distances, hO QO ApJ 457 (500) 1996

[38] S. A. Sim, F. K.Ropke, W.Hillebrandt, M.Kromer, R.Pakmor, M.Fink, A. J. Ruiter,J.R. Seitenzahl, Detonations in sub-Chandrasekhar mass $C+O$ white dwarfs ApJ 714 (52) 2010 
[39] J. Guillochon, M. Dan, E. Ramirez-Ruiz, S. Rosswog, Surface detonations in double degenerate systems triggered by accretion stream inestabilities ApJ 709 (64) 2010

[40] K. J.Shen, L. Bildsten, Unstable helium shell burning on accreting white dwarfs ApJ 699 (1365) 2009

[41] D. R. Poznanski et al., An unusually fast evolving supernova Science 327 (58) 2010

[42] H. B. Perets et al, A faint type of supernova from a white dwarf with a helium-rich companion Nature 465 (322) 2010

[43] I. P.Pskovskii, Light curves, color curves and expansion velocity of type I supernovae as functions of the rate of brghtness decline Sov. Astron 21 (675) 1977

[44] M. M. Phillips, The absolute magnitudes of Type Ia supernovae ApJ 413 (L105) 1993

[45] I. L. Prieto, A. Rest, N. B.Suntzeff, A new method to calibrate the magnitudeds of Type Ia supernovae at maximum light ApJ 647 (501) 2006

[46] D. Kasen, S. E.Woosley, On the origin of the Type Ia supernova width-luminosity relationship ApJ 656 (661) 2007

[47] S. E. Woosley, D. Kasen, S. Blinnikov, E. Sorokina, Type Ia supernova light curves ApJ 662 (487) 2007

[48] P. A.Mazzali, F. K.Ropke, S. Benetti, W. Hillebrandt, A common explosion mechanism for type Ia supernovae Science 315 (825)

[49] E. Bravo, J. Isern, R. Canal, J. Labay, On the contribution of Ne-22 to the synthesis of $\mathrm{Fe}-54$ and $\mathrm{Ni-58}$ in thermonuclear supernovae A\&A 257 (534) 1992

[50] E. Bravo, A. Tornambe, I. Dominguez, J.Isern, Clues to Type Ia supernova progenitors from degenerate carbon ignition models A\&A 306 (811) 1996

[51] E. Bravo, I. Dominguez, J. Isern, R. Canal, P. Hoflich, J.Labay, On the photometric homogeneity of type Ia supernovae A\&A 269 (187) 1993

[52] W. D. Arnett, Type I supernovae. I-Analytic solutions for the early part of the light curve ApJ 253 (785) 1982

[53] D. D. Clayton, S. A.Colgate, G. J. Fishman, Gamma-ray lines from young supernova remnants ApJ 155 (75) 1969

[54] A. Burrows, L. The, X-and gamma-ray signatures of type Ia supernovae ApJ 360 (626) 1990

[55] S. Kumagai, K.Nomoto, Gamma-rays and X-rays from type Ia supernovae in Ruiz-Lapuente P.,Canal,R., Isern J. (Eds) NATO ASIC Proc. 486: Thermonuclear Supernovae (515) 1997

[56] J.Gomez-Gomar, J. Isern, P. Jean, Prospects for type Ia supernova mechanism identification with gamma-rays MNRAS 295 (1) 1998

[57] P. A. Milne et al., Unifies one-dimensional simulation of gamma-ray emission from type Ia supernovae ApJ 613 (1101) 2004

[58] J. Isern, E. Bravo, A. Hirschmann, Detection and interpretation of gamma-ray emission from SNIa New Astr.Rev. 52 (377) 2008 